\section{Fuerza prensil de mano y su asociación con la edad, género y dominancia de extremidad superior en adultos mayores autovalentes insertos en la comunidad. Un estudio exploratorio}

\author{
FRANCISCO GUEDE ROJAS ${ }^{1, \mathrm{a}}$, LUIS JAVIER CHIROSA RÍOS ${ }^{2, \mathrm{~b}}$, \\ CÉSAR VERGARA RÍOS ${ }^{1, \mathrm{c}}$, JORGE FUENTES CONTRERAS ${ }^{3, \mathrm{~d}}$, \\ FRANCISCO DELGADO PAREDES ${ }^{1, \mathrm{e}}$, \\ MARÍA JOSÉ VALDERRAMA CAMPOS ${ }^{1, \mathrm{c}}$
}

\section{Association of grip strength with gender age and handedness in 116 older people}

Background: Hand grip strength could be influenced by gender, age and handedness. Aim: To describe differences in grip strength for age, gender and upper extremity handedness in non-disabled community-dwelling older adults. Material and Methods: We studied 47 males aged $72.3 \pm 5.6$ years and 69 females $72.4 \pm 6.0$ years who were divided in two age groups (65-70 and $\geq 71$ years old). Grip strength was determined by a standardized protocol using a hand dynamometer. Results: There was an inverse correlation between grip strength and age in both hands only among men $(p \leq 0.05)$. When analyzing both genders, there was a significant inverse correlation between grip strength and age only in the dominant hand ( $p \leq$ $0.05)$. Strength was higher in the dominant hand in both genders $(p \leq 0.05)$. It was also higher in men, compared to women in the two age groups studied ( $p \leq 0.05)$. Conclusions: Grip strength is higher in men than women, it decreases with age and is higher in the dominant hand.

(Rev Med Chile 2015; 143: 995-1000)

Key words: Aged; Functional laterality; Gender; Geriatric Assessment; Hand Strength.

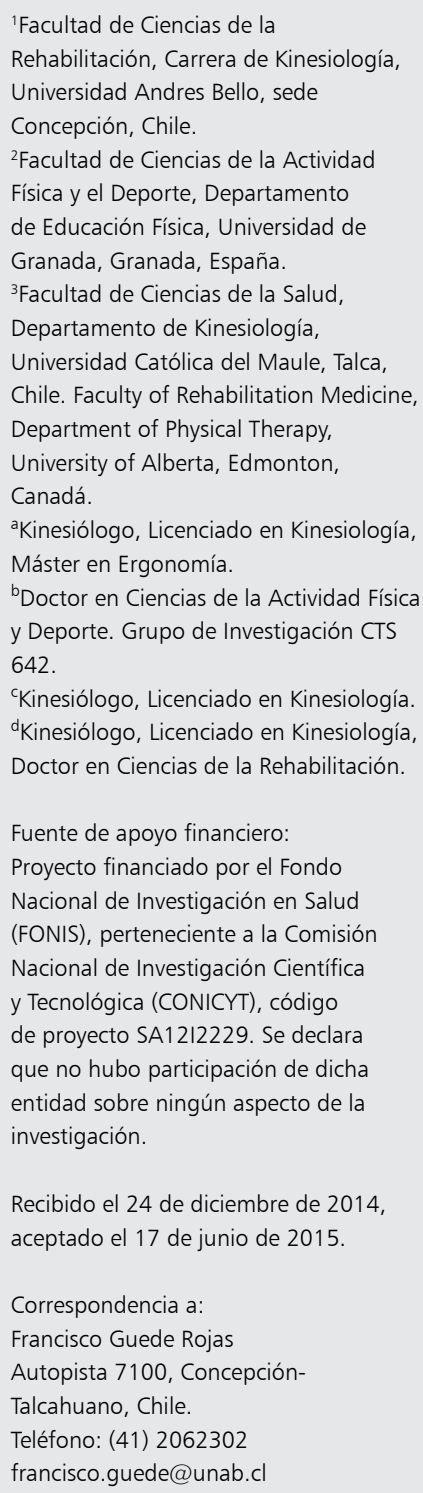

'Kinesiólogo, Licenciado en Kinesiología.

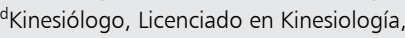
Doctor en Ciencias de la Rehabilitación.

Fuente de apoyo financiero: Proyecto financiado por el Fondo Nacional de Investigación en Salud (FONIS), perteneciente a la Comisión Nacional de Investigación Científica y Tecnológica (CONICYT), código de proyecto SA1212229. Se declara que no hubo participación de dicha entidad sobre ningún aspecto de la investigación.

Recibido el 24 de diciembre de 2014, aceptado el 17 de junio de 2015.

Correspondencia a:

Francisco Guede Rojas

Autopista 7100, Concepción-

Talcahuano, Chile.

Teléfono: (41) 2062302

francisco.guede@unab.cl

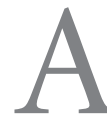

nivel mundial existe una transición demográfica hacia el envejecimiento y Chile no escapa de esta realidad, estimándose que para el año 2025 la proporción de adultos mayores (AM) será de $20,1 \%$ de la población total ${ }^{1}$. El envejecimiento corresponde a un proceso natural complejo que involucra cambios físicos, psicológicos y sociales que pueden influir negativamente sobre la calidad de vida del $\mathrm{AM}^{2}$. Dentro de los cambios físicos más relevantes se encuentra la reducción progresiva de las capacidades que definen la condición física del AM destacando la fuerza muscular ${ }^{3}$, la cual puede verse afectada por múltiples factores asociados a la reducción de masa muscular, como trastornos endocrinos, neurodegenerativos y nutricionales, junto con la inactividad física y el sedentarismo $\mathrm{o}^{4-6}$. La reducción de masa y función muscular conocida con el nombre de sarcopenia, se ha asociado negativamente con el desempeño físico y movilidad, determinando mayor depen- 
dencia funcional, discapacidad física y aumento de la morbimortalidad en esta población ${ }^{5,7,8}$.

De acuerdo al consenso del Grupo de Trabajo Europeo sobre Sarcopenia en Personas Mayores (EWGSOP), la medición de la fuerza prensil de mano (FPM) constituye el método más simple y recomendado para la evaluación de la fuerza muscular en la práctica clínica, debido a su fuerte asociación con la fuerza muscular de las extremidades inferiores y el área de sección transversal muscular de la pantorrilla ${ }^{8}$. La FPM puede ser determinada midiendo la fuerza isométrica máxima que la mano genera alrededor de un dinamómetro y se expresa generalmente en kilogramos, pondios, milímetros de mercurio y newtons ${ }^{9}$. La dinamometría de mano es una medición confiable y válida cuando se utilizan métodos estandarizados y equipos calibrados ${ }^{10-12}$.

Se ha planteado la asociación entre la FPM y diversas condiciones de salud del $\mathrm{AM}^{13}$, siendo reconocida como un indicador que puede ayudar a identificar personas en riesgo de limitación funcional $^{14}$, fragilidad física y discapacidad ${ }^{15}$. Además, corresponde a un factor predictor importante de caídas y morbilidad después de intervenciones quirúrgicas, dentro de otros aspectos ${ }^{16}$. Los datos normativos publicados de FPM usualmente se expresan en función de rangos etarios y género, no existiendo un consenso en cuanto a otras categorizaciones como la lateralidad y dominancia de la extremidad superior (DES $)^{9,17-19}$. En términos generales, se sostiene que la FPM es consistentemente mayor en hombres que en mujeres ${ }^{9-19}$, que los niveles máximos de fuerza se alcanzan dentro de la cuarta década de vida ${ }^{20}$ y que sujetos diestros son más fuertes con su mano derecha, mientras que los zurdos presentan resultados controversiales ${ }^{21}$. De igual manera, existen estudios que analizan el comportamiento de la FPM en función de la edad, describiendo una fuerte relación inversa FPMedad, la cual es más significativa que la relación inversa FPM-masa muscular ${ }^{22}$ y más acentuada en hombres de edad avanzada ${ }^{23}$.

Si bien la FPM corresponde a un indicador predictor de salud y funcionalidad del AM, utilizado además para la evaluación de diversas condiciones relacionadas especialmente con disfunciones de la mano, la literatura plantea que sus valores difieren significativamente entre poblaciones $y$ que por tanto la utilización de datos normativos internacionales es cuestionable ${ }^{24}$. En Chile, existen estudios que describen la FPM y su relación con la funcionalidad ${ }^{25} \mathrm{y}$ estado nutricional en $\mathrm{AM}^{26}$. Sin embargo, a pesar de su amplio respaldo científico la información disponible en nuestro país es escasa y no se encuentran antecedentes que analicen la relación FPM-edad en función del género y DES. Además, no se encuentran antecedentes que describan los niveles de FPM en función de categorías etarias de AM. Por tanto, a partir de los antecedentes disponibles y reconociendo la relevancia clínica de la FPM, el propósito del presente estudio piloto fue determinar la relación FPM-edad en función del género y DES, así como también, determinar diferencias de FPM de acuerdo a la edad, género y DES en una muestra de AM autovalentes insertos en la comunidad.

\section{Materiales y Métodos}

\section{a) Diseño y reclutamiento de casos}

Estudio descriptivo exploratorio de corte transversal en el que participaron $116 \mathrm{AM}$, de los cuales 47 (40,5\%) eran hombres y 69 (59,5\%) mujeres. Los sujetos debían presentar una clasificación funcional de "Autovalente" de acuerdo con el Examen Funcional del Adulto Mayor (EFAM) y el examen Mini Mental Status Examination (MMSE) abreviado, además de contar con certificación médica para participar del estudio. Se excluyeron sujetos con ceguera e hipoacusia severa, amputación de extremidades superiores, secuelados de accidente vascular encefálico y presentar cualquier condición física o mental que pudiese atentar contra la adecuada realización de las pruebas o ser incapaces de finalizarlas. Todos los sujetos pertenecían al Centro Comunitario de Salud Familiar (CECOSF) "Libertad-Gaete" ubicado en la comuna de Talcahuano, VIII región Chile y representaron 26,4\% del total de AM autovalentes inscritos en el CECOSF.

El presente estudio forma parte de un proyecto financiado por FONIS-CONICYT y todos los procedimientos fueron aprobados por el Comité de Bioética de la Universidad Andrés Bello. Los sujetos fueron contactados y citados en grupos a una charla informativa acerca de los objetivos y requerimientos del estudio. Los sujetos que accedieron a participar firmaron voluntariamente un consentimiento informado y fueron citados consecuentemente a una segunda fase donde 
se les realizó un chequeo médico, completaron un formulario de información general y se les administró las pruebas correspondientes. Todos los procedimientos fueron realizados en las dependencias del CECOSF "Libertad-Gaete" entre abril y agosto de 2013.

\section{b) Instrumento y protocolo}

Para la evaluación de la FPM se utilizó un dinamómetro hidráulico Jamar ${ }^{\circledR}$ (PC 5030 J1, Sammons Preston Rolyan, EE. UU). La ejecución de la prueba se basó en los criterios establecidos por la American Society of Hand Therapists (ASHT) ${ }^{27}$. Los sujetos adoptaron la posición sedente con el brazo aducido, el codo flexionado a $90^{\circ}$ y la muñeca neutral. Se sostuvo el dinamómetro en posición II con garra cilíndrica mientras el evaluador lo apoya ligeramente desde la base. Se le solicitó a los sujetos realizar tres esfuerzos de prensión rápidamente progresivos hasta alcanzar el máximo posible con una pausa de 30 segundos entre cada uno de ellos. Seguidamente se registró el mayor valor en kilogramos tanto para la FPM dominante (FPMD) como para la FPM no-dominante (FPMND).

\section{c) Análisis estadístico}

Los resultados se describieron en términos de media aritmética y desviación estándar. La verificación del supuesto de normalidad de los datos se llevó a cabo mediante la prueba $\mathrm{W}$ de Shapiro-Wilk y la verificación del supuesto de homogeneidad de varianzas mediante la prueba F de Levene. Para analizar los datos se indagó sobre la existencia de relaciones entre las variables FPMD-FPMND y la edad mediante la prueba rho de Spearman. Para la comparación entre la FPMD y FPMND en hombres y mujeres de acuerdo al rango etario se utilizó la prueba $t$ de muestras relacionadas y para realizar la comparación de FPM entre hombres y mujeres de acuerdo a DES y rango etario se utilizó la prueba t de muestras no relacionadas. Los datos registrados de cada una de las variables del estudio se procesaron mediante el software InfoStat versión 2013, utilizando un nivel de significación de 5\%.

\section{Resultados}

\section{Sujetos}

Las características demográficas de la muestra se exponen en la Tabla 1. Del grupo de hombres, $23(48,9 \%)$ estaban en el rango de 65 a 70 años y $24(51,1 \%)$ tenían 71 años o más. Por su parte, de las mujeres $31(44,9 \%)$ estaban en el rango de 65 a 70 años y $38(55,1 \%)$ tenían 71 años o más. La edad promedio de la muestra fue de $72,4 \pm 5,9$ años y la proporción de diestros fue de $95,7 \%$ en hombres y de $100 \%$ en mujeres.

\section{Relación FPM-edad}

La relación FPM-edad se muestra para hombres y mujeres en la Tabla 2. En hombres, la relación fue inversa y estadísticamente significativa tanto en la mano dominante como no-dominante $(\mathrm{p} \leq 0,05)$. Mientras que en mujeres, las relaciones inversas no fueron significativas en ninguna de las dos manos $(\mathrm{p} \leq 0,05)$.

Los resultados de la relación FPM-edad según DES al considerar la muestra completa de hombres

Tabla 1. Descripción demográfica de la muestra de estudio

\begin{tabular}{|c|c|c|c|c|c|c|}
\hline \multirow[b]{2}{*}{$\begin{array}{l}\text { Parámetros } \\
\text { demográficos }\end{array}$} & \multicolumn{2}{|c|}{ Grupo 65-70 años } & \multicolumn{2}{|c|}{ Grupo $\geq 71$ años } & \multirow{2}{*}{$\begin{array}{l}\text { Total } \\
\text { Hombres } \\
(n=47)\end{array}$} & \multirow[b]{2}{*}{$\begin{array}{l}\text { Mujeres } \\
(n=69)\end{array}$} \\
\hline & $\begin{array}{l}\text { Hombres } \\
(n=23)\end{array}$ & $\begin{array}{l}\text { Mujeres } \\
(n=31)\end{array}$ & $\begin{array}{l}\text { Hombres } \\
(n=24)\end{array}$ & $\begin{array}{l}\text { Mujeres } \\
(n=38)\end{array}$ & & \\
\hline Edad (años) & $67,6 \pm 1,6$ & $67,6 \pm 1,8$ & $76,8 \pm 4,3$ & $76,3 \pm 5,4$ & $72,3 \pm 5,6$ & $72,4 \pm 6,0$ \\
\hline Peso $(\mathrm{Kg})$ & $81,3 \pm 16,0$ & $73,7 \pm 17,3$ & $72,4 \pm 14,3$ & $69,7 \pm 15,2$ & $76,8 \pm 15,6$ & $71,5 \pm 16,2$ \\
\hline Talla (mts) & $1,6 \pm 0,1$ & $1,5 \pm 0,1$ & $1,6 \pm 0,1$ & $1,5 \pm 0,1$ & $1,6 \pm 0,1$ & $1,5 \pm 0,1$ \\
\hline $\mathrm{IMC}\left(\mathrm{Kg} / \mathrm{mts}^{2}\right)$ & $30,4 \pm 4,8$ & $32,7 \pm 6,2$ & $28,5 \pm 5,1$ & $31,1 \pm 6,0$ & $29,5 \pm 5,0$ & $31,8 \pm 6,1$ \\
\hline Diestros (\%) & 91,7 & 100 & 100 & 100 & 95,7 & 100 \\
\hline Zurdos (\%) & 8,3 & 0 & 0 & 0 & 4,3 & 0 \\
\hline
\end{tabular}

IMC: Índice de masa corporal. 
Tabla 2. Resultados sobre la relación FPM-edad según género y dominancia

\begin{tabular}{|llcc|}
\hline \multirow{2}{*}{ Edad } & rho de Spearman & $\begin{array}{c}\text { FPMD } \\
\text { hombres }\end{array}$ & $\begin{array}{c}\text { FPMND } \\
\text { hombres }\end{array}$ \\
\cline { 2 - 4 } & Valor-p & $-0,45$ & $-0,43$ \\
& & $0,001 *$ & $0,002^{*}$ \\
\multirow{2}{*}{ Edad } & rho de Spearman & FPMD & FPMND \\
& Valor-p & $-0,23$ & $-0,00$ \\
& & 0,060 & 0,998 \\
\hline
\end{tabular}

FPMD: Fuerza prensil de mano dominante. FPMND: Fuerza prensil de mano no-dominante. * Correlación estadísticamente significativa ( $p \leq 0,05)$.

y mujeres, se presenta en la Tabla 3. Se observa que la relación FPMD-edad es inversa y estadísticamente significativa a diferencia de la relación FPMND-edad, en la cual la relación inversa no es significativa $(\mathrm{p} \leq 0,05)$.

\section{Comparación de la FPM entre la mano dominante y no-dominante}

En la Tabla 4 se presenta la comparación entre la FPMD y FPMND en función del género y rango etario. Para el grupo completo tanto de hombres como mujeres, la FPMD fue mayor $(\mathrm{p} \leq 0,05)$. Al considerar los géneros en función de los rangos etarios, solamente las mujeres entre 65 a 70 años presentaron mayor FPMD ( $\mathrm{p} \leq 0,05)$. Los hombres presentaron mayores niveles de fuerza que las mujeres tanto en la mano dominante como no-dominante $(\mathrm{p} \leq 0,05)$.

\section{Discusión}

La FPM puede ser afectada por múltiples factores vinculados a la disminución de la masa muscular ${ }^{4-6}$, correspondiendo a un indicador relevante asociado a la salud y funcionalidad del $\mathrm{AM}^{13-16}$. Existe información relativa a los niveles de FPM en función a la edad, género y DES $S^{9,17,18,19}$, así como también de la relación inversa FPMedad $^{22,23}$. Sin embargo, los datos normativos son controversiales y pueden diferir significativamente entre poblaciones ${ }^{24,28}$. Por tanto, el propósito de este estudio fue describir el comportamiento de la FPM en función de la edad, género y DES en una muestra de AM chilenos autovalentes.
Tabla 3. Resultados de la relación de FPM-edad en función de la dominancia para toda la muestra

\begin{tabular}{|llll|}
\hline \multirow{2}{*}{ Edad } & rho de Spearman & $-0,24$ & $-0,16$ \\
& Valor-p & $0,008^{*}$ & 0,079 \\
\hline
\end{tabular}

FPMD: Fuerza prensil de mano dominante. FPMND: Fuerza prensil de mano no-dominante. *Correlación estadísticamente significativa $(p \leq 0,05)$.

Tabla 4. Comparación entre FPMD y FPMND en hombres y mujeres en función del rango etario

\begin{tabular}{|llccl|}
\hline & & $\begin{array}{c}\text { FPMD } \\
(\mathbf{k g})\end{array}$ & $\begin{array}{c}\text { FPMND } \\
(\mathbf{k g})\end{array}$ & $\begin{array}{c}\text { Valor } \\
\text { p }\end{array}$ \\
\hline $\begin{array}{l}\text { 65-70 } \\
\text { años }\end{array}$ & Hombres & $35,6 \pm 7,2$ & $34,2 \pm 8,9$ & 0,30 \\
& Mujeres & $22,5 \pm 8,0$ & $19,6 \pm 7,8$ & $0,00^{*}$ \\
años & Hombres & $30,9 \pm 6,4$ & $29,7 \pm 6,6$ & 0,10 \\
Total & Mujeres & $19,4 \pm 7,0$ & $19,4 \pm 5,7$ & 0,93 \\
& Mombres & $33,1 \pm 7,0$ & $31,5 \pm 8,1$ & $0,05^{*}$ \\
\hline
\end{tabular}

FPMD: Fuerza prensil de mano dominante. FPMND: Fuerza prensil de mano no-dominante. *Diferencia estadísticamente significativa $(p \leq 0,05)$.

Los resultados aportados por esta investigación establecen que sólo en el caso de los hombres la relación FPM-edad es inversa y estadísticamente significativa, tanto en la mano dominante como no-dominante, lo cual sugiere que la proporción de reducción de FPM es más acentuada en este grupo. Estos resultados son concordantes con los reportados por Desrosiers et $\mathrm{al}^{23}$, quienes describieron una mayor relación inversa FPM-edad en hombres comparado con mujeres. Esta situación podría atribuirse a que los hombres, al alcanzar mayores niveles de FPM durante la vida adulta estarían más propensos a sufrir caídas más acentuadas en sus niveles de fuerza como consecuencia de cambios en sus estilos de vida y de las menores exigencias físicas propias de la adultez mayor. Por su parte, la ausencia de significación en la relación FPM-edad en el grupo de mujeres podría deberse a que en general ellas no modificarían sustancialmente sus actividades regulares, presentando niveles más homogéneos de fuerza a lo largo de 
su vida. En discordancia a estos resultados, Daly et $\mathrm{a}^{20}$, en un estudio prospectivo de 10 años no reportaron diferencias por género en cuanto a tasa de disminución de la FPM en AM.

En el presente estudio, cuando ambos géneros fueron considerados, la relación FPMD-edad fue inversa y estadísticamente significativa, mientras que la relación FPMND-edad no lo fue. Este resultado sugiere que la tasa de reducción de FPMD es mayor que la tasa de reducción de FPMND en función de la edad.

La literatura plantea que la mano dominante presenta mayores niveles de fuerza que la mano no-dominante y se asume que esta diferencia puede llegar a ser alrededor de $10 \%$ en personas diestras ${ }^{29}$. Al respecto, el presente estudio fue conformado predominantemente por personas diestras y al considerar las muestras totales de hombres y mujeres se confirmó esta noción. Sin embargo, al analizar las diferencias por grupos etarios, éstas no fueron significativas con excepción del grupo de mujeres con edades entre 65 y 70 años.

Se ha descrito ampliamente que los hombres presentan mayor FPM que las mujeres ${ }^{9,17,18}$. En este sentido, recientemente Yorke et $\mathrm{al}^{19}$ describieron para la FPM derecha, que en el rango de 60 a 69 años las medias fueron de $41 \mathrm{~kg}$ y $25 \mathrm{~kg}$ para hombres y mujeres respectivamente, mientras que en el rango de 70 a 79 años, las medias fueron de 36 $\mathrm{kg}$ y $21 \mathrm{~kg}$ respectivamente. En el presente estudio, si bien las características de los sujetos, los rangos etarios y los niveles de FPM no son comparables a los de estudios previos, también se observa que los hombres presentaron consistentemente mayor FPM que las mujeres en las dos categorías etarias consideradas y en ambas manos.

Evidencia disponible valida la FPM como un indicador de función muscular asociada a autonomía y calidad de vida del $\mathrm{AM}^{30}$, así como también, su relación con otros indicadores y predictores de salud $^{13}$. A pesar de estas características positivas y su relativo bajo costo, en nuestro país esta prueba no se recomienda en el Examen de Medicina Preventiva del AM (EMPAM) ${ }^{31}$ y actualmente su uso no se encuentra ampliamente difundido. De igual manera, escasos estudios nacionales se han focalizado en la descripción de la FPM y su relación con diversos aspectos de salud del $\mathrm{AM}^{25,26}$.

Aunque los resultados obtenidos de esta investigación son interesantes y corresponden a antecedentes preliminares asociados al comportamiento de la FPM en AM chilenos, su limitación es la acotada validez externa o posibilidad de generalización de ellos, debido a que sólo se incluyeron AM de un centro comunitario de la comuna de Talcahuano y es posible que los valores de FPM difieran en poblaciones provenientes de otras comunas de la VIII región u otras regiones del país.

En conclusión, los hallazgos de esta investigación confirman que en la muestra de estudio, la edad, género y DES influyen sobre la FPM. Los hombres presentan mayores niveles de FPM que las mujeres y presentan una mayor reducción de FPM con la edad. La FPMD es mayor que la FPMND y se reduce de manera más acentuada con la edad.

Agradecimientos: Los autores agradecen a CONICYT-FONIS, a la Dirección de Administración en Salud (DAS) de la Ilustre Municipalidad de Talcahuano y al personal del CECOSF "Libertad Gaete" por el apoyo que hizo posible la realización de este estudio.

\section{Referencias}

1. Instituto Nacional de Estadística (INE). (2008). Población y sociedad. Aspectos demográficos. Disponible en http://www.ine.cl/canales/chile_estadistico/demografia_y_vitales/demografia/pdf/poblacion_sociedad_enero09.pdf [Consultado el 22 de marzo de 2015].

2. Bauer J, Sieber C. Sarcopenia and frailty: a clinician's controversial point of view. Exp Gerontol 2008; 43: 6748.

3. Bohannon R, Magasi S. Identification of dynapedia in older adults through the use of grip stregth t-scores. Muscle Nerve 2015; 51: 102-5.

4. Paddon-Jones D, Short K, Campbell W, Volpi E, Wolfe $\mathrm{R}$. Role of dietary protein in the sarcopenia of aging. Am J Clin Nutr 2008; 87: 1562S-6S.

5. Thompson D. Aging and sarcopenia. J Musculoskelet Neuronal Interact 2007; 7 (4): 344-5.

6. Nelson M, Rejeski W, Blair S, Duncan P, Judge J, King A, et al. Physical Activity and Public Health in Older Adults: Recommendation From the American College of Sports Medicine and the American Heart Association. Circulation 2007; 116 (9): 1094-105.

7. Rantanen T, Harris T, Leveille S. Muscle Strength and Body Mass Index as Long Term Predictors of Mortality in Initially Healthy Men. J Gerontol A Biol Sci Med Sci 2000; 55: M168-73. 
8. Cruz-Jentoft A, Baeyens J, Bauer J, Boirie Y, Cederholm T, Landi F, et al. Sarcopenia: European consensus on definition and diagnosis: Report of the European Working Group on Sarcopenia in Older People. Age Ageing 2010; 39 (4): 412-23.

9. Massy-Westropp N, Gill T, Taylor A, Bohannon R, Hill C. Hand Grip Strength: Age and gender stratified normative data in a population-based study. BMC Research Notes 2011; 4: 127-31.

10. Mathiowetz M. Comparison of Rolyan and Jamar dynamometers for measuring grip strength. Occ Ther Int 2002; 9 (3): 201-9.

11. Schmidt N, van der Windt D, Assendelft W, Mourits A, Deville W, de Winter A, et al. Interobserver Reproducibility of the Assessment of Severity of Complaints, Grip Strength, and Pressure Pain Threshold in Patients With Lateral Epicondylitis. Arch Phys Med Rehabil 2002; 83 (8): 1145-50.

12. Mijnarends D, Meijers J, Halfens R, Borg S, Luiking Y, Verlaan S, et al. Validity and Reliability of Tools to Measure Muscle Mass, Strength, and Physical Performance in Community-Dwelling Older People: A Systematic Review. JAMDA 2013; 14 (3): 170-8.

13. Cooper R, Kuh D, Cooper C, Gale C, Lawlor D, Matthews $\mathrm{F}$, et al. Objective measures of physical capability and subsequent health: a systematic review. Age and Ageing 2011; 40 (1): 14-23.

14. Sallinen J, Stenholm S, Rantanen T, Heliövaara M, Sainio P, Koskinen S. Hand-grip strength cut points to screen older persons at risk for mobility limitation. J Am Geriatr Soc 2010; 58 (9): 1721-6.

15. Syddall H, Cooper C, Martin F, Briggs B, Saye A. Is grip strength a useful single marker of frailty? Age and ageing 2003; 32 (6): 650-6.

16. Angst F, Drerup S, Goldhahn J. Prediction of grip and key pinch strength in 978 healthy subjects. BMC Musculoskelet Disord 2010; 11: 94-9.

17. Mathiowetz V, Kashman N, Volland G, Weber K, Dowe M, Rogers S. Grip and pinch strength: normative data for adults. Arch Phys Med Rehabil 1985; 66 (2): 69-74.

18. Bohannon R, Peolsson A, Massy-Westropp N, Desrosiers J, Bear-Lehman J. Reference values for adult grip strength measured with a Jamar dynamometer: a descriptive meta-analysis. Physiotherapy 2006; 92 (1): 11-5.

19. Yorke A, Curtis A, Shoemaker M, Vangsnes E. Grip Strength Values Stratified by Age, Gender, and Chronic Disease Status in Adults Aged 50 Years and Older. J Geriatr Phys Ther 2015; 00: 1-7.
20. Daly R, Rosengren B, Alwis G, Ahlborg H, Sernbo I, Karlsson M. Gender specific age-related changes in bone density, muscle strength and functional performance in the elderly: a-10 year prospective population-based study. BMC Geriatrics 2013; 13: 71-9.

21. Bohannon R. Grip strength: a summary of studies comparing dominant and nondominant limb measurements. Percept Mot Skills 2003; 96 (1): 728-30.

22. Kallman D, Plato C, Tobin J. The Role of Muscle Loss in the Age-Related Decline of Grip Strength: Cross-Sectional and Longitudinal Perspectives. Journal Of Gerontology 1990; 45 (3): M82-8.

23. Desrosiers J, Bravo G, Hebert R, Dutil E. Normative Data for Grip Strength of Elderly Men and Women. American Journal of Occupational Therapy 1995; 49 (7): 637-44.

24. Werle S, Goldhahn J, Drerup S, Simmen B, Sprott H, Herren D. Age-and gender-specific normative data of grip and pinch strength in a healthy adult swiss population. The Journal of Hand Surgery 2009; 34E (1): 76-84.

25. Arroyo P, Lera L, Sánchez H, Bunout D, Santos J, Albala C. Indicadores antropométricos, composición corporal y limitaciones funcionales en ancianos. Rev Med Chile 2007; 135 (7): 846-54.

26. Pino J, Mardones M, Díaz C. Relación entre la dinamometría de mano y la circunferencia de pantorrilla con el índice de masa corporal en ancianos autovalentes. Rev Chil Nutr 2011; 38 (1): 23-9.

27. Fess E. Grip strength. In Clinical assessment recommendations. 2 edition. Editorial Casanova JS. Chicago: ASHT 1992; 41-5.

28. Roberts H, Denison H, Martin H, Patel H, Syddall H, Cooper $\mathrm{C}$, et al. A review of the measurement of grip strength in clinical and epidemiological studies: towards a standardised approach. Age and Ageing 2011; 40 (4): 423-9.

29. Crosby C, Wehbe M, Mawr B. Hand strength: Normative values. Journal of Hand Surgery 1994; 19A: 665-70.

30. Sayer A, Syddall H, Martin H, Dennison E, Roberts H, Cooper C. Is grip strength associated with health-related quality of life? Findings from the Hertfordshire Cohort Study. Age and Ageing 2006; 35 (4): 409-15.

31. Ministerio de Salud (MINSAL). Gobierno de Chile. Manual de Aplicación del Examen de Medicina Preventiva del Adulto Mayor EMPAM. Disponible en http://web. minsal.cl/sites/default/files/files/PDF\%20Examen $\% 20$ de\%20Medicina \%20Preventiva\%20del\%20Adulto\%20 Mayor.pdf [Consultado el 13 de octubre de 2014]. 